\title{
The RNA transport factor PHAX is required for proper histone H2AX expression and DNA damage response
}

\author{
MITSUHIRO MACHITANI, ${ }^{1,2}$ ICHIRO TANIGUCHI, ${ }^{1}$ ASAKO MCCLOSKEY, ${ }^{1}$ TATSUYA SUZUKI, ${ }^{1}$ \\ and MUTSUHITO OHNO ${ }^{1}$ \\ ${ }^{1}$ Institute for Frontier Life and Medical Sciences, Kyoto University, Sakyo-ku, Kyoto 606-8507, Japan \\ ${ }^{2}$ Division of Cancer Stem Cell, National Cancer Center Research Institute, Tokyo 104-0045, Japan
}

\begin{abstract}
PHAX (phosphorylated adaptor for RNA export) promotes nuclear export of short transcripts of RNA polymerase II such as spliceosomal U snRNA precursors, as well as intranuclear transport of small nucleolar RNAs (snoRNAs). However, it remains unknown whether PHAX has other critical functions. Here we show that PHAX is required for efficient DNA damage response (DDR) via regulation of phosphorylated histone variant H2AX ( $\gamma \mathrm{H} 2 A X)$, a key factor for DDR. Knockdown of PHAX led to a significant reduction of H2AX mRNA levels, through inhibition of both transcription of the H2AX gene and nuclear export of H2AX mRNA, one of the shortest mRNAs in the cell. As a result, PHAX-knockdown cells become more sensitive to DNA damage due to a shortage of $\gamma \mathrm{H} 2 \mathrm{AX}$. These results reveal a novel function of PHAX, which secures efficient DDR and hence genome stability.
\end{abstract}

Keywords: PHAX; histone mRNA; H2AX; DNA damage response

\section{INTRODUCTION}

DNA lesions, caused by environmental stresses such as DNA injuring chemicals, ultraviolet (UV) light, ionizing radiation, etc., rapidly induce DNA damage response (DDR) that involves DNA repair, cell cycle arrest, and apoptosis, leading to the maintenance of genome integrity and tissue homeostasis (Jackson and Bartek 2009). DNA lesions activate protein kinases such as ataxia telangiectasia mutated (ATM) kinase, Rad3-related (ATR) kinase, and DNA-dependent protein kinase, catalytic subunit (DNA-PKcs), which gather to the DNA damage sites and phosphorylate histone variant H2AX at Ser139 (Thiriet and Hayes 2005). The resultant phosphorylated $\mathrm{H} 2 \mathrm{AX}(\gamma \mathrm{H} 2 \mathrm{AX})$ forms "DDR foci" on the injured chromatin sites, which function as platforms for recruiting other DDR effectors (Jackson and Bartek 2009). $\gamma \mathrm{H} 2 \mathrm{AX}$-mediated formation of DDR foci is a mark of the early step of the DDR pathway and is critical for efficient DDR. The H2AX protein turns over rapidly in normal situations, but is stabilized by DNA damage (Atsumi et al. 2015).

To focus on the topic of this study, it is necessary to describe an apparently unrelated field, RNA export. We have previously shown that nuclear export of the spliceosomal U snRNA precursors requires two adaptor proteins,

Corresponding author: hitoohno@infront.kyoto-u.ac.jp

Article is online at http://www.rnajournal.org/cgi/doi/10.1261/rna. 074625.120. the cap-binding complex (CBC) and the phosphorylated adaptor for RNA export (PHAX) as well as CRM1, the export receptor for proteins containing a leucine-rich nuclear export signal and RanGTP (Ohno et al. 2000; Will and Luhrmann 2001). PHAX and CRM1 were also implicated in an intranuclear transport of small nucleolar RNAs (snoRNAs) (Boulon et al. 2004). We have subsequently shown that RNA polymerase II (RNAPII) transcripts are sorted according to their lengths (Ohno et al. 2002; Masuyama et al. 2004; McCloskey et al. 2012). The RNAPII transcripts shorter than 200-300 nt are funneled into the spliceosomal $U$ snRNA export pathway involving PHAX and CRM1, while the transcripts longer than the threshold are funneled into the mRNA export pathway involving mRNA export factors, including the TREX components and the export receptor NXF1/TAP but excluding PHAX. Notably, mRNAs for histones including H2AX are the shortest mRNAs in the cells and their lengths are quite close to the above threshold. In addition, the majority of histone mRNAs are produced without splicing and polyadenylation as U snRNAs (Marzluff et al. 2008). Hence histone mRNAs are unique mRNAs resembling $U$ snRNAs.

(c) 2020 Machitani et al. This article is distributed exclusively by the RNA Society for the first 12 months after the full-issue publication date (see http://rnajournal.cshlp.org/site/misc/terms.xhtml). After 12 months, it is available under a Creative Commons License (Attribution-NonCommercial 4.0 International), as described at http:// creativecommons.org/licenses/by-nc/4.0/. 
Although it has been shown that nuclear export of histone mRNAs requires mRNA export factors (Erkmann et al. 2005), the requirement for $U$ snRNA export factors such as PHAX has not been investigated.

In this study, we found that knockdown of PHAX led to a significant reduction in H2AX expression levels, through inhibitions of H2AX mRNA export as well as of H2AX promoter activity. In response to DNA damage, PHAX-knockdown cells failed to induce efficient DNA repair due to a shortage of $\gamma H 2 A X$. These results reveal a novel function of PHAX, which secures efficient DDR and hence genome stability via regulation of histone $\mathrm{H} 2 \mathrm{AX}$ expression.

\section{RESULTS}

\section{PHAX knockdown leads to a reduction in tolerance to DNA damage}

During our survey process for undocumented functions of PHAX, we examined cell viability upon stimulation with anti-cancer agents and UV. We found that PHAX is involved in the cell viability after stimulation causing DNA damage. In the experiments, U2OS cell was used because of its wild-type p53 expression. The viability of PHAX-knockdown (KD) U2OS cells was markedly reduced after UV irradiation as compared to that of the control cells (Fig. 1A, lower panels), whereas cell viability without UV irradiation was not significantly altered (Fig. 1A, upper panels). Similar results were obtained with the cells treated by anti-cancer drugs such as adriamycin (ADR, Fig. 1B) or camptothecin (CPT, Fig. 1C). Notably, the UV irradiation-mediated inductions of p53 and phosphorylated p53 were enhanced in PHAX-KD cells (Fig. 1D).

Since these results suggested that DDR may be disturbed in PHAX-KD cells, DNA repair efficiency in these cells was next examined. It is known that two major repairs, homologous-dependent repair (HDR) and nonhomologous end joining (NHEJ), are induced after double-strand DNA breaks (DSBs) (Shrivastav et al. 2008). PHAX-KD or control-KD cells were transfected with the reporter plasmids for each DNA repair assay (pDRGFP for HDR and
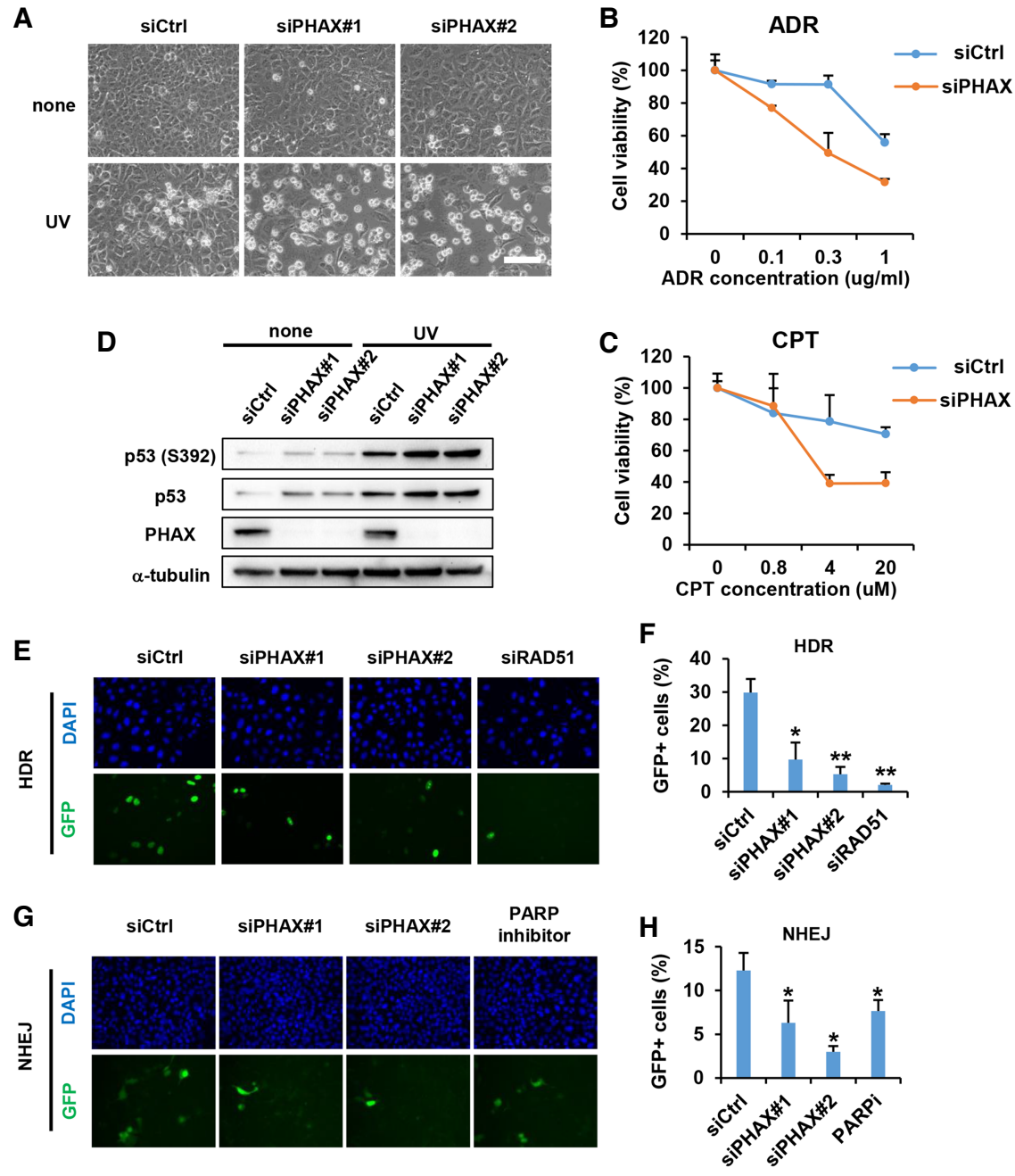

FIGURE 1. Inhibition of DNA repair by knockdown of PHAX. (A) U2OS cells were transfected with the indicated siRNAs, followed by UV-irradiation $\left(20 \mathrm{~J} / \mathrm{m}^{2}\right)$. After 6-h incubation, phasecontrast images were obtained. $(B, C)$ U2OS cells were transfected with the indicated siRNAs, followed by ADR (B) or CPT (C) at the indicated concentrations. After 24-h incubation, cell viabilities were determined by alamarBlue assay. (D) U2OS cells were treated as in $A$. The cotransfected with the indicated siRNAs and each reporter plasmid for DNA repair assay ( were obtained, and the numbers of GFP-positive cells were counted. As a positive control, the cells were treated with PARP inhibitor (PARPi) at $100 \mathrm{nM}$ for $48 \mathrm{~h}$. Data are the means \pm S.D. $(n=4) .\left(^{*}\right) P<0.01,\left(^{* *}\right) P<0.001$.

pEJ2GFP for NHEJ, respectively), and the two DNA repair assays were performed (see Supplemental Fig. S1A for the HDR assay scheme). PHAX-KD cells showed a significant decrease in HDR efficiency as compared to the control cells (Fig. 1E,F; Supplemental Fig. S1B,C). This decrease was quite comparable to that of the positive controls, RAD51$\mathrm{KD}$ cells (Fig. 1E,F) and H2AX-KD cells (Supplemental Fig. S1B,C; Shrivastav et al. 2008; Jackson and Bartek 2009). Efficiencies of NHEJ were also suppressed by over $50 \%$ in PHAX-KD cells (Fig. 1G,H). The effect of PHAX$\mathrm{KD}$ was comparable to the positive control cells that had been treated by a PARP inhibitor, olaparib (Fig. 1G,H). 
Note that there was no difference in transfection efficiency among the reporter plasmids under the experimental conditions (data not shown). Taken together, these results indicate that PHAX is required for efficient DSB repair.

\section{PHAX knockdown leads to a reduction in $\mathrm{H} 2 \mathrm{AX}$ expression}

To clarify the cause of the inefficient DDR in PHAX-KD cells, we next examined the expression levels of several DDR factors. PHAX-KD promoted the phosphorylation levels of ATM and DNA-PKcs after ADR treatment, while $\gamma \mathrm{H} 2 \mathrm{AX}$ was not efficiently induced in PHAX-KD cells (Fig. 2A,B). Immunofluorescence cell staining analyses also demonstrated that the induction of $\gamma \mathrm{H} 2 \mathrm{AX}$ was inhibited in PHAX-KD cells (Fig. 2C). Similar results were obtained in UV-irradiated cells (Fig. 2D). When the PHAX-KD cells were transfected with a PHAX-expressing plasmid, the $\gamma \mathrm{H} 2 \mathrm{AX}$ expression level was largely restored (Fig. 2D), indicating that it is PHAX that promotes $\gamma \mathrm{H} 2 \mathrm{AX}$ expression.

To examine the cause of the low $\gamma \mathrm{H} 2 \mathrm{AX}$ expression, the total H2AX protein expression level was examined by using an antibody against $H 2 A X$, which recognizes both phosphorylated and unphosphorylated H2AX proteins. After treatment of the cells with UV or ADR, the expression levels of both total and phosphorylated $\mathrm{H} 2 \mathrm{AX}$ proteins were lower in PHAX-KD cells as compared to those in the control cells, although their levels were constantly low without UV or ADR (Fig. 2E). These results demonstrated that PHAX-KD cells were not able to induce efficient H2AX protein expression and hence efficient DNA damage repair, because reduction of $\mathrm{H} 2 \mathrm{AX}$ expression in fact leads to reduction in DNA repair efficiency (Supplemental Fig. S1B,C).

To examine why H2AX protein expression is low, we next measured mRNA levels of $\mathrm{H} 2 \mathrm{AX}$ by quantitative reverse-transcription PCR (qRT-PCR) analysis. Regardless of the DNA damage induction, the steady state $\mathrm{H} 2 \mathrm{AX}$ mRNA level was reduced by $~ 50 \%$ in PHAX-KD cells, as compared to control-KD cells (Fig. 3A). In addition, mRNA levels of various replicationdependent histones were also reduced by half in PHAX-KD cells (Fig. $3 B$ ). In contrast, expression of replication-dependent histone mRNAs, but not H2AX mRNA, was heavily depressed by the DNA damage induction probably due to a DNA replication arrest, consistent with a previous report (Dankert et al. 2016). Therefore it was not possible to determine the effect of PHAX-KD (data not shown). The reduction of $\mathrm{H} 2 \mathrm{~A}$ and H2AX mRNA levels was restored by a rescue of $\mathrm{PHAX}$ expression, at least partially (Fig. 3C, Supplemental Fig. S2A), indicating that it is PHAX that is critical for this phenomenon.

PHAX-KD had specific suppressive effects on histone gene expressions among the genes tested (Fig. 3D), although c-myc expression was enhanced for unknown reasons. Note that steady state $U$ snRNA levels were not significantly altered by PHAX-KD despite PHAX's role as a $U$ snRNA exporter (Fig. 3D; Supplemental Fig. S2B) likely because U snRNAs are very stable in the cell. This indicates that the reduction in histone mRNA levels in PHAX-KD cells was 

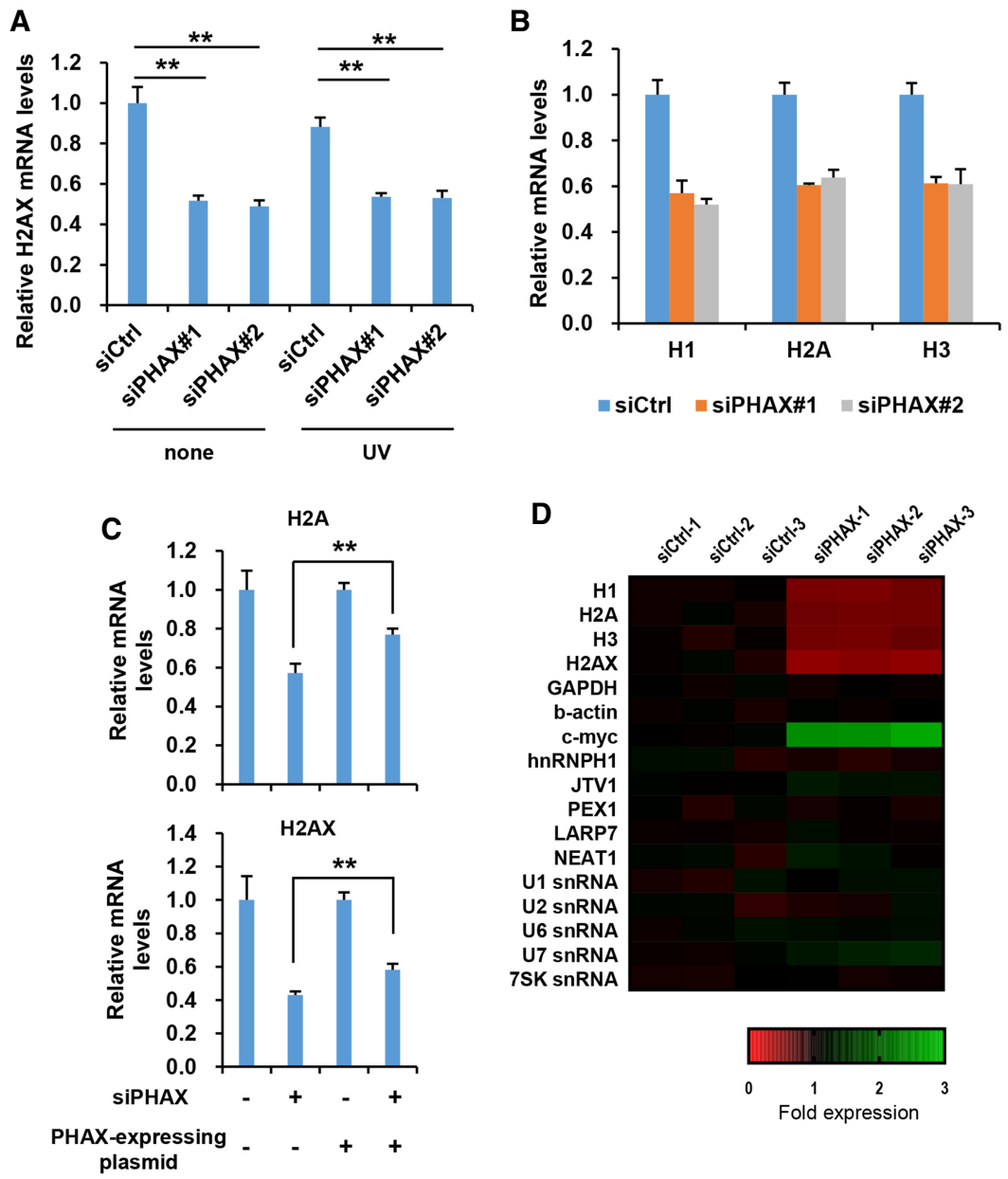

FIGURE 3. Reduction in H2AX mRNA levels by knockdown of PHAX. (A) U2OS cells were transfected with the indicated siRNAs, followed by UV-irradiation $\left(20 \mathrm{~J} / \mathrm{m}^{2}\right)$. After 6 -h incubation, the indicated mRNA levels were determined by qRT-PCR analysis. (B) U2OS cells were transfected with the indicated siRNAs. After 48-h incubation, the indicated mRNA levels were similarly determined. (C) U2OS cells were cotransfected with siPHAX and a PHAX-expressing plasmid. After 48-h incubation, the indicated mRNA levels were similarly determined. (D) The indicated mRNA levels in siPHAX-transfected cells were determined by qRT-PCR analysis and were expressed in the form of a heat map. Data are the means \pm S.D. $\left.(n=3-4) .{ }^{* *}\right) P<$ 0.001 .

not attributed to the interruption of $U$ snRNA biogenesis and/or splicing.

PHAX accumulates in Cajal bodies (CBs), and the CBs are depleted when expression of PHAX is suppressed in cells (Lemm et al. 2006). In addition, UV treatment causes $\mathrm{CB}$ disassembly that is mediated by a proteasome activator PA28 $\gamma$, and overexpression of PA28 $\gamma$ causes depletion of CBs even in the cells not treated by UV (Cioce et al. 2006). To examine whether CBs affect histone mRNA expression, we induced depletion of $\mathrm{CBs}$ in the cell by an overexpression of PA28 $\gamma$ (Cioce et al. 2006). In USOS cells overexpressing PA28 $\gamma$, CBs were depleted (Supplemental Fig. S2C), but histone mRNA levels were not significantly altered (Supplemental Fig. S2D), suggesting that it was not $C B$ depletion that affected histone mRNA levels.
Since replication-dependent histone mRNAs, such as $\mathrm{H} 1, \mathrm{H} 2 \mathrm{~A}, \mathrm{H} 2 \mathrm{~B}$, $\mathrm{H} 3$, and $\mathrm{H} 4$, are transcribed during $\mathrm{S}$ phase (Marzluff et al. 2008), we also examined the cell cycle property of PHAX-KD cells. The KD of PHAX had no great effects on the cell cycle property, except that the cell population in G1 and S phase was only slightly increased and decreased, respectively (Supplemental Fig. S2E), indicating that the reduction in replication-dependent histone mRNA levels in PHAX-KD cells cannot be attributed to a disturbance of cell cycle. To further examine the effect of cell cycle property on histone gene expression, we synchronized the cell cycle in G1 phase by a double-thymidine block. Expression of replication-dependent histone mRNAs depends on cell cycle, while H2AX mRNA expression does not. Even in the condition of cell synchronization in G1 phase, H2AX mRNA expression was significantly decreased in PHAX-KD cells (Supplemental Fig. S2F), suggesting that $\mathrm{PHAX}$ regulates $\mathrm{H} 2 \mathrm{AX}$ expression independently of cell cycle property.

We have so far shown that steady state mRNA levels of histones including $\mathrm{H} 2 \mathrm{AX}$ are reduced by $\sim 50 \%$ in PHAX-KD cells (Fig. 3A). It is worth pointing out that the protein levels of histones including H2AX are not significantly altered in normal situations without DNA damage induction (Fig. 2E, Supplemental Fig. S2G) but that the protein expression level of H2AX is heavily suppressed in DNA damaging conditions, probably due to the stabilization of H2AX protein in DNA damaging conditions (Fig. 2E).

\section{PHAX knockdown leads to reductions in histone gene transcription}

In order to examine whether PHAX is involved in histone mRNA expression at early steps such as transcription and RNA processing, histone mRNA levels during early steps were analyzed in PHAX-KD cells. RNA was pulse-labeled by 5-ethynyl uridine (5EU) for $1.5 \mathrm{~h}$, and purified through an immunoprecipitation by an anti-5EU antibody. qRT$P C R$ analyses demonstrated that the nascent mRNA levels of H2A, H3, and H2AX but not GAPDH, were significantly decreased in PHAX-KD cells (Fig. 4A), suggesting that 


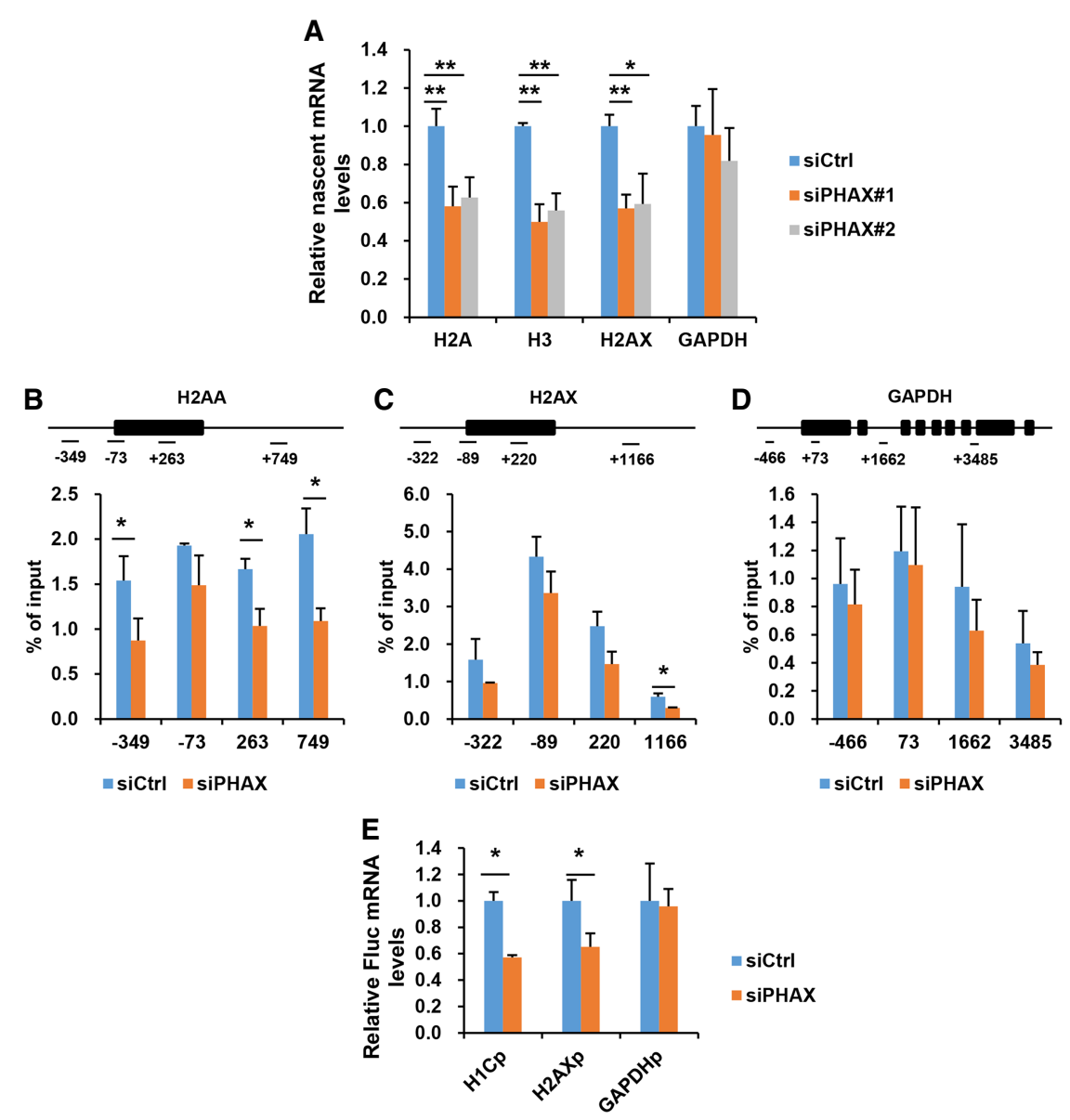

FIGURE 4. Suppression of histone mRNA transcription by knockdown of PHAX. (A) U2OS cells were transfected with the indicated siRNAs. After 48-h incubation, nascent RNAs were labeled by $5 E$ for $1.5 \mathrm{~h}$. 5EU-labeled nascent mRNAs were determined by GRT-PCR analysis. (B-D) U2OS cells were transfected with siPHAX. After 48-h incubation, RNAPII-binding DNA levels on the indicated gene loci were determined by ChIP assay. (E) U2OS cells were cotransfected with siPHAX, an Rluc-expressing plasmid (pGL4-SV40p-Rluc), and the reporter plasmid carrying $\mathrm{HIC}, \mathrm{H} 2 \mathrm{AX}$, or GAPDH promoter-driven Fluc expression cassette. After 48-h incubation, Fluc mRNA levels were determined by qRT-PCR analysis. Fluc mRNA levels were normalized by Rluc mRNA levels. Data are the means \pm S.D. $(n=3-4) .\left({ }^{*}\right) P<0.05,\left({ }^{* *}\right) P<0.01$. tion of replication-dependent histone mRNAs is driven by a transcriptional regulator NPAT (nuclear protein, ataxia-telangiectasia locus), which is recruited to histone gene clusters and forms a nuclear structure called the histone locus body (HLB) in the form of nuclear dots (Marzluff et al. 2008). The HLBs were detected in both control and PHAX-KD cells (Supplemental Fig. S3A), suggesting that PHAXKD had no effects on the expression of NPAT and the formation of HLBs.

It was possible that PHAX KD might affect RNA stabilization. A previous report has demonstrated that PHAX and an exosome adaptor $\mathrm{ZC} 3 \mathrm{H} 18$ are mutually exclusive during interaction with CBC-bound RNAs, and that in PHAX-KD cells, ZC3H18 is preferentially associated with CBC-bound RNAs, leading to destabilization of the RNAs (Giacometti et al. 2017). To examine the involvement of $\mathrm{ZC} 3 \mathrm{H} 18$, ZC3H18 was knocked down simultaneously with PHAX. The expression of $\mathrm{ZC} 3 \mathrm{H} 18$ proteins was successfully knocked down after transfection with siRNA against ZC3H18 (siZC3H18) (Supplemental Fig. S3B). Histone mRNA levels in PHAX/ZC3H18 double-KD (DKD) cells were not significantly altered as compared to those in PHAX-KD cells (Supplemental Fig. $\mathrm{S} 3 \mathrm{C})$, suggesting that $\mathrm{ZC} 3 \mathrm{H} 18$ was not involved in the reduction in histone mRNA expression in PHAX-KD cells. histone mRNA transcription was partially inhibited by PHAX-KD. Chromatin Immunoprecipitation (ChIP) analyses revealed that RNAPII recruitments to $H 2 A A$ and H2AX gene loci were reduced in PHAX-KD cells, as compared to those in control cells (Fig. 4B-D). Note that RNAPII recruitments to the promoter regions (H2AA: -349; H2AX: -322) were attenuated (Fig. 4B,C). This prompted us to examine the activity of histone gene promoters using reporter plasmids in which the promoter sequences from $\mathrm{H} 1 \mathrm{C}$ and $\mathrm{H} 2 \mathrm{AX}$ were fused to the coding sequence of the firefly luciferase (Fluc). The reporter assay confirmed that the promoter activities of $\mathrm{H} 1 \mathrm{C}$ and $\mathrm{H} 2 \mathrm{AX}$ were significantly decreased by PHAX-KD (Fig. 4E). These results indicate that $\mathrm{PHAX}$ regulates the transcription of the histone genes. The molecular mechanism for the transcription regulation is currently unknown. It is known that the transcrip-

\section{PHAX knockdown leads to a reduction of histone mRNA export}

PHAX plays an important role in nuclear export of short transcripts of RNAPII including spliceosomal U snRNA precursors. Since histone mRNAs are the shortest mRNAs in the cell, we next examined whether PHAX is also involved in histone mRNA export. To this end, a tagged H2A- or H2AX-expressing plasmid was transfected into PHAX-KD or control-KD cells. As controls, U1 $\Delta$ Sm RNA-, or EGFP-expressing plasmid was also transfected. U1 $1 \mathrm{Sm}$ RNA is a mutant U1 snRNA capable of export but not reimport (Hamm and Mattaj 1990). After incubation, the cells were harvested, fractionated into the cytoplasmic and the nuclear fractions, and the export efficiency (the cytoplasmic to nuclear ratio) of the RNAs transcribed from the transfected 
plasmids as well as endogenous GAPDH mRNA were analyzed. The purity of each fraction was confirmed by analyzing endogenous NEAT1 RNA (mainly localized in the nucleus) and endogenous GAPDH mRNA levels (mainly localized in the cytoplasm) (Supplemental Fig. S4A). The export efficiency of $\mathrm{H} 2 \mathrm{~A}$ and $\mathrm{H} 2 \mathrm{AX}$ mRNAs, as well as $\mathrm{U} 1 \Delta \mathrm{Sm}$ RNA, but not EGFP and GAPDH mRNAs, was significantly decreased by PHAX-KD (Fig. 5A), while the export of all mRNAs but not of U1 $\triangle \mathrm{Sm}$ RNA was severely reduced by NXF1/TAP-KD (Supplemental Fig. S4B,C; Erkmann et al. 2005), suggesting that efficient export of histone mRNAs requires PHAX on top of mRNA export machinery. Consistent with this is PHAX's association with various histone mRNAs as well as U1 snRNA but not GAPDH and $\beta$ actin mRNAs, as shown by immunoprecipitation analyses (Fig. 5B). To further examine whether PHAX is required for histone mRNA export in a different experimental system, a precursor of human $\mathrm{H} 2 \mathrm{~A}$ mRNA was microinjected into the nucleus of Xenopus oocytes (Fig. 5C). U6Ass RNA which has a small deletion in the single-stranded re-
A

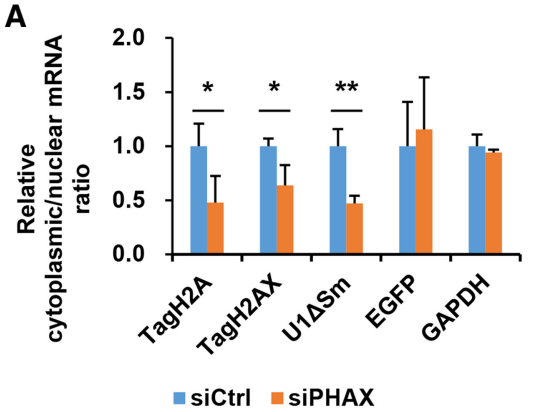

C

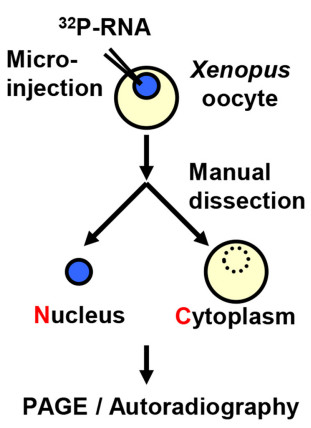

B

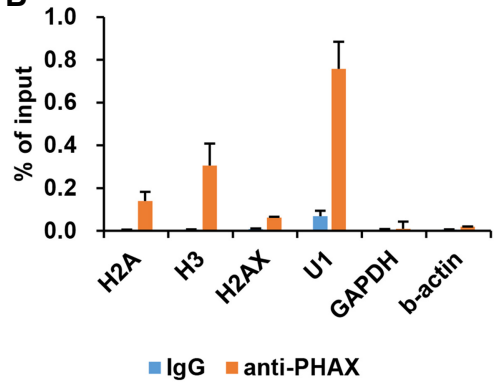

D

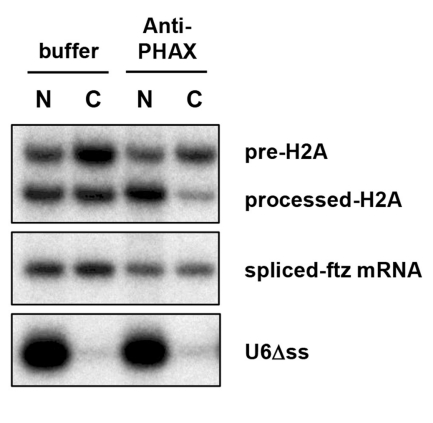

FIGURE 5. Reduction in histone mRNA export efficiencies by knockdown of PHAX. (A) U2OS cells were transfected with siPHAX, followed by transfection with plasmids expressing the indicated genes. After 3-h incubation, RNA was extracted from the nuclear and cytoplasmic fractions of the cells, and the indicated mRNA levels were determined by qRT-PCR analysis. (B) U2OS cells were lysed and immunoprecipitation was performed with an anti-PHAX antibody. PHAX-binding RNA levels were determined by qRT-PCR analysis. $(C, D) A$ mixture of ${ }^{32} \mathrm{P}$-labeled m7G-capped precursor $\mathrm{H} 2 \mathrm{~A}$ (pre-H2A), precursor ftz mRNA, and U6 $\Delta$ ss was injected into the nucleus of Xenopus oocytes either alone or together with an anti-PHAX antibody. RNA was extracted from nuclear $(\mathrm{N})$ and cytoplasmic $(\mathrm{C})$ fractions $2 \mathrm{~h}$ after microinjection and analyzed by $8 \%$ denaturing PAGE followed by autoradiography. Pre- $\mathrm{H} 2 \mathrm{~A}$ was processed into mature $\mathrm{H} 2 \mathrm{~A}$ (shown as processed-H2A) and pre-ftz mRNA was spliced (spliced-ftz mRNA) in the nucleus. Data are the means \pm S.D. $(n=3) .\left({ }^{*}\right) P<0.05,\left({ }^{* *}\right) P<0.01$. gion was used as a marker for the efficacy of nuclear microinjection, because the U6 $\Delta$ ss RNA is not imported into the nucleus if misinjected into the cytoplasm (Hamm and Mattaj 1990). The precursor H2A mRNA was processed to produce mature H2A mRNA after microinjection (Fig 5D). Microinjection of the antibody against PHAX inhibited the export of processed H2A mRNA but not the control RNA (Fig. 5D). These results confirm that PHAX regulates DISCUSSION

In this study, we have clarified a previously undocumented role of RNA transport factor PHAX. We have provided evdence showing that PHAX regulates histone H2AX expression and DDR. Since PHAX is required for efficient H2AX mRNA synthesis and nuclear export, PHAX-KD cell cannot efficiently induce $\gamma \mathrm{H} 2 \mathrm{AX}$ expression (Fig. 2) and hence DDR (Fig. 1) when DNA is damaged, due to a shortage of H2AX protein. Steady state mRNA levels of H2AX are modestly reduced (by $\sim 50 \%$ ) in PHAX-KD cells (Fig. 3A). This modest reduction of H2AX mRNA leads to a large reduction of the H2AX protein level, not in normal situations but in DNA damaging situations (Fig. 2). This is probably because H2AX protein rapidly turns over in the former situations but is highly stabilized in the latter (Atsumi et al. 2015). In addition, our data show that PHAX also regulates the production of mRNAs for replication-dependent histones similarly at the levels of both transcription (Fig. 4) and mRNA export (Fig. 5).

Although our results clearly indicate that $\mathrm{PHAX}$ regulates the transcription of histone genes quite specifically, the molecular mechanism for the transcription regulation is currently unknown. As shown in Figure 4B, the RNAPII recruitment to the promoter region is as high as that to the transcriptional start sites (TSSs), especially in the case of H2AA, although RNAPII usually stalls at TSS (Ji et al. 2013). This suggests that transcription of histone mRNAs might be progressed promptly without stalling of RNAP ॥ at TSSs, and that the RNAPII recruitment to the promoter region might be a rate-limiting step. Recruitment of RNAPII to histone gene loci and their promoter activities were shown to be reduced in PHAX-KD cells (Fig. 
4), suggesting that PHAX could affect these steps. Furthermore, it is interesting that transcription of both replication-dependent histones and -independent histone $\mathrm{H} 2 \mathrm{AX}$ is affected, since their transcription regulations are quite different. This suggests that PHAX may be involved in the basic transcription machinery common to all histone genes. PHAX is recruited to $\mathrm{CBC}$ together with an adaptor protein ARS2 that directly interact with FLASH (Kiriyama et al. 2009). FLASH is known to regulate histone gene transcription through a direct interaction with NPAT (Marzluff et al. 2008). Integrity of HLBs and their constituent NPAT are critical for the transcription of replication-dependent histones such as $\mathrm{H} 1, \mathrm{H} 2 \mathrm{~A}, \mathrm{H} 2 \mathrm{~B}, \mathrm{H} 3$, and $\mathrm{H} 4$ (Zhao et al. 2000; Marzluff et al. 2008). Nonetheless, we have at least shown that HLBs are not affected by PHAX-KD (Supplemental Fig. S3A). Further investigation of how PHAX is involved in the transcription of a variety of histone genes is necessary.

In $U$ snRNA export, PHAX is recruited to $C B C$ that has been bound to the cap structure and the recruited PHAX in turn recruits the export receptor CRM1 and RanGTP, leading to the RNA export to the cytoplasm (Ohno et al. 2000). In contrast, bulk mRNAs are bound by adaptor proteins including the TREX components and/or SR proteins and these adaptors in turn recruit the export receptor NXF1/TAP, leading to the bulk mRNA export to the cytoplasm (Kohler and Hurt 2007). Notably, the U snRNA export factors such as PHAX are usually excluded from the mRNA export complex (Masuyama et al. 2004). We have previously shown that the RNAPII transcripts shorter than 200-300 nt are funneled into U snRNA export pathway involving PHAX and CRM1, while the transcripts longer than the threshold are funneled into the mRNA export pathway involving mRNA export factors, including the TREX components and NXF1/TAP (McCloskey et al. 2012). Although histone mRNAs are the shortest mRNAs in the cells and their lengths are quite close to the above threshold, it has been already demonstrated that the histone mRNA export is carried out by NXF1/TAP-, but not CRM1-, mediated export pathway (Erkmann et al. 2005). Consistent with a previous study (Giacometti et al. 2017), our results demonstrated that PHAX is recruited to histone mRNAs unlike the bulk mRNAs (Fig. 5B) and that PHAX is required for the efficient nuclear export of histone mRNAs on top of the mRNA export factors (Fig. 5A,D). The effect of PHAX$\mathrm{KD}$ in this process is relatively modest as compared to that of the NXF1/TAP-KD (Fig. 5A; Supplemental Fig. $\mathrm{S} 4 \mathrm{C})$. Therefore, it seems that PHAX plays only an optimizing role in the nuclear export of histone mRNAs. However, this modest decrease in mRNA export together with a modest decrease in gene transcription does lead to a large reduction in $\gamma \mathrm{H} 2 \mathrm{AX}$ expression in DNA damage situations. How PHAX plays a role in the mRNA export of histone mRNAs is currently unknown. Only what we know is that PHAX seems to be a part of the histone mRNA export complex (Fig. 5B) but that CRM1 seems not involved unlike in the case of $U$ snRNA export (Erkmann et al. 2005). Further studies will be needed to clarify the mechanism underlying PHAX-mediated export of histone mRNAs.

Although there have already been several reports describing roles of RNA binding proteins in DDR (Dominguez-Sanchez et al. 2011; Adamson et al. 2012; Awwad et al. 2017), our unexpected results described here further reveal how the mechanism promoting the maintenance of genome integrity is multifarious and extensive.

\section{MATERIALS AND METHODS}

\section{Cells, chemicals, and antibodies}

U2OS cells (a human osteosarcoma cell line) were cultured in Dulbecco's modified Eagle's medium supplemented with $10 \% \mathrm{fe}-$ tal bovine serum (FBS), streptomycin $(100 \mu \mathrm{g} / \mathrm{mL})$, and penicillin (100 U/mL). ADR (also known as doxorubicin), CPT, and PARPi (Olaparib) were purchased from FUJIFILM Wako Pure Chemical Corp., Sigma Aldrich Japan, and Selleckchem, respectively. The antibodies used in this study are described in Supplemental Table S1.

\section{Plasmids}

pDRGFP (plasmid 26475), pEJ2GFP (44025), pCBAScel (26477) were obtained from Addgene. Human PHAX cDNA containing FLAG-tag was amplified by PCR from a HeLa cDNA library and cloned into pcDNA3, resulting in pcDNA3-FLAG-PHAX. The sequences of the primers used in this study are described in Supplemental Table S2.

The reporter plasmids pGL4-H1Cp, -H2AXp, and -GAPDHp, which have sequences from the respective promoters upstream of the Fluc gene, were constructed as follows: The fragment containing each promoter was amplified by PCR using the corresponding primers and cloned into pGL4.10 (Promega). pGL4SV40p-Rluc, a reporter plasmid carrying a SV40 promoter-driven renilla luciferase (Rluc) expression cassette, was purchased from Promega (sold as pGL4.73).

The fragment containing human $\mathrm{H} 2 \mathrm{~A}$ promoter and coding region was amplified by PCR from HeLa genomic DNA and cloned into pUC118, resulting in pUC118-hH2Ap-hH2A. To insert tag sequence (5'-TGATCGCGCTTCTCGTTG-3'), site-directed mutagenesis of pUC118-hH2Ap-hH2A was performed using appropriate primers, resulting in pUC118-hH2Ap-tag hH2A. A plasmid carrying human $\mathrm{H} 2 \mathrm{AX}$ promoter and coding region, pUC118-hH2AXp-tag hH2AX, was similarly constructed. The sequences of the primers used in this study are described in Supplemental Table S2.

\section{Transfection with siRNA}

All siRNAs (Stealth siRNA of the 25-mer duplex) used in this study were obtained from Thermo Fisher Scientific. Cells were 
transfected with the siRNAs using Lipofectamine2000 (Thermo Fisher Scientific) according to the manufacturer's instructions.

\section{qRT-PCR analysis}

Total RNA was isolated from cells using Sepasol-RNA I Super (Nacalai Tesque). cDNA was synthesized using $500 \mathrm{ng}$ of total RNA with a Superscript VILO cDNA Synthesis Kit (Thermo Fisher Scientific). qRT-PCR analysis was performed using Fast SYBR Green Master Mix (Thermo Fisher Scientific) and StepOnePlus Real-Time PCR systems (Thermo Fisher Scientific). All data of qRT-PCR analysis were normalized by the data of GAPDH mRNA levels. The sequences of the primers used in this study are described in Supplemental Table S2.

\section{Western blotting analysis}

Western blotting assay was performed as previously described (Machitani et al. 2020). Briefly, whole-cell extracts were prepared and $10 \mu \mathrm{g}$ of total protein per lane was loaded onto $10 \%$ sodium dodecyl sulfate (SDS)-polyacrylamide gels. After electrophoresis under reducing conditions, bands of protein were transferred to polyvinylidene difluoride (PVDF) membranes (Merck Millipore). After blocking with $5 \%$ skim milk prepared in TBS-T (Tween$20,0.1 \%$ ), the membrane was incubated with the primary antibodies, followed by incubation in the presence of horseradish peroxidase (HRP)-labeled anti-mouse or -rabbit lgG antibody (Jackson ImmunoResearch). The antibodies used in this study are described in Supplemental Table S1. The intensities of protein bands were quantified using Image $\mathrm{J}$ software.

\section{Immunofluorescence cell staining}

Immunofluorescence cell staining was performed as previously described (Izumi et al. 2014). Briefly, cells were fixed with $4 \%$ formaldehyde in PBS, permeabilized with $0.2 \%$ TritonX-100 in PBS, and blocked with $2 \%$ bovine serum albumin in PBS. The cells were incubated with the primary antibodies, followed by incubation in the presence of Alexa488-, Alexa568-, or Cy5-labeled secondary antibody (Thermo Fisher Scientific). The antibodies used in this study are described in Supplemental Table S1.

\section{DNA repair assays}

DNA repair assay was performed as previously described (Seluanov et al. 2010). Cells were cotransfected with pDRGFP (for HDR) or pEJ2GFP (for NHEJ) and pCBAScel using Lipofectamine2000 (Thermo Fisher Scientific) according to the manufacturer's instructions. After 48- or 72-h incubation, fluorescence microscopic images were obtained, and the numbers of GFP-positive cells were counted.

\section{Cell viability assay}

For the evaluation of cell viability after UV treatment, cells were irradiated with UV $\left(20 \mathrm{~J} / \mathrm{m}^{2}\right)$. After 6-h incubation, phase-contrast photomicrographs of the cells were obtained.
For the evaluation of the cytotoxic activity of ADR and CPT, cells were treated with ADR or CPT at the indicated concentrations. After 24-h incubation, the cell viabilities were determined by staining with alamarBlue (Thermo Fisher Scientific) according to the manufacturer's instructions.

\section{Determination of nascent RNA levels}

U2OS cells were transfected with the indicated siRNAs. After 48-h incubation, nascent RNAs were labeled by $5 E U$ at 0.5 $\mathrm{mM}$ for $1.5 \mathrm{~h}$. 5EU-labeled RNAs were biotinylated and immunoprecipitated using Click-it Nascent RNA Capture Kit (Thermo Fisher Scientific) according to the manufacturer's instructions. The nascent RNA levels were determined by qRTPCR analysis.

\section{Chromatin immunoprecipitation (ChIP) assay}

U2OS cells were transfected with siRNAs. After 48-h incubation, cells were treated with formaldehyde at a final concentration of $1 \%$ for crosslinking, and then genomic DNA was fragmented by sonication. The DNA fragment-protein complexes were immunoprecipitated using a mouse anti-RNA polymerase II CTD antibody (8WG16) (Abcam). The ChIP assay kit was purchased from Merck Millipore. The precipitated DNA copy numbers were determined by quantitative PCR analysis using the primers shown in Supplemental Table S2.

\section{RNA immunoprecipitation experiments}

RNA immunoprecipitation experiments were performed as previously described (McCloskey et al. 2012). Briefly, for formaldehyde crosslinking, cells were treated with formaldehyde at a final concentration of $1 \%$. The reaction was quenched by adding a final $0.15 \mathrm{M}$ glycine. After cells were washed with icecold PBS, cell pellets were resuspended in RIPA buffer. Cell lysate was prepared by sonication with Bioruptor (CosmoBio). After centrifugation, PHAX-binding RNA was immunoprecipitated using a mouse anti-PHAX antibody from the lysate. The precipitated RNA copy numbers were determined by qRT-PCR analysis.

\section{Nuclear and cytoplasmic mRNA fractionation}

U2OS cells were transfected with siPHAX, followed by transfection with tagged-H2A, - H2AX, $-U 1 \Delta S m$ (Izumi et al. 2014), or EGFP-expressing plasmids (pUC118-hH2Ap-taghH2A, -hH2AXp-taghH2AX, hU1p-hU1 $\Delta$ Sm, or pEGFP-C1 [Clontech], respectively). After 3-h incubation, the nuclear and cytoplasmic fractions of the cells were prepared using NE-PE Nuclear and Cytoplasmic Extraction Reagents (Thermo Fisher Scientific) according to the manufacturer's instructions. RNA was isolated from each fraction as described above, and the indicated mRNA levels were determined by qRT-PCR analysis.

\section{Microinjection into Xenopus oocytes}

For the human $\mathrm{H} 2 \mathrm{~A}$ plasmid, the human $\mathrm{H} 2 \mathrm{~A}$ fragment was amplified by PCR from a HeLa cDNA library and cloned into pBluescript 
$\mathrm{KS}(+)$, resulting in pBS-hH2A. pBS-hH2A was linearized by Notl, followed by transcription by T7 RNA polymerase. ${ }^{32} \mathrm{P}$-labeled U6 $\Delta$ ss RNA and precursor $\mathrm{ftz}$ mRNA were prepared as described previously (Ohno et al. 2000). Microinjection of the ${ }^{32}$ P-labeled RNAs into Xenopus oocytes was performed as previously described (Ohno et al. 2000). Nuclear and cytoplasmic RNA was recovered at $2 \mathrm{~h}$ after microinjection.

\section{Statistical analysis}

Statistical significance was determined using Student's t-test. Data are presented as the means \pm S.D.

\section{SUPPLEMENTAL MATERIAL}

Supplemental material is available for this article.

\section{ACKNOWLEDGMENTS}

We thank the members of our laboratory, especially Makoto Kitabatake, for many useful suggestions and criticisms of the manuscript. This work was supported by a Grant-in-Aid for Scientific Research (A) (no. 25251004) and for Scientific Research on Innovative Areas "Non-coding RNA Neo-taxonomy" (no. 26113004) from the Ministry of Education, Culture, Sports, Science and Technology (MEXT) of Japan.

Author contributions: M.M. designed and performed the experiments, analyzed data, and wrote the manuscript; I.T. performed and supported the experiments; T.S. and A.M. performed some founding old experiments; M.O. obtained the funding, designed some experiments, supervised the projects, and wrote the manuscript.

Received January 1, 2020; accepted August 2, 2020.

\section{REFERENCES}

Adamson B, Smogorzewska A, Sigoillot FD, King RW, Elledge SJ. 2012. A genome-wide homologous recombination screen identifies the RNA-binding protein RBMX as a component of the DNA-damage response. Nat Cell Biol 14: 318-328. doi:10.1038/ ncb2426

Atsumi Y, Minakawa Y, Ono M, Dobashi S, Shinohe K, Shinohara A, Takeda S, Takagi M, Takamatsu N, Nakagama H, et al. 2015. ATM and SIRT6/SNF2H mediate transient H2AX stabilization when DSBs form by blocking HUWE1 to allow efficient $\gamma \mathrm{H} 2 \mathrm{AX}$ foci formation. Cell Rep 13: 2728-2740. doi:10.1016/j.celrep .2015 .11 .054

Awwad SW, Abu-Zhayia ER, Guttmann-Raviv N, Ayoub N. 2017. NELF-E is recruited to DNA double-strand break sites to promote transcriptional repression and repair. EMBO Rep 18: 745-764. doi:10.15252/embr.201643191

Boulon S, Verheggen C, Jady BE, Girard C, Pescia C, Paul C, Ospina JK, Kiss T, Matera AG, Bordonne R, et al. 2004. PHAX and CRM1 are required sequentially to transport U3 snoRNA to nucleoli. Mol Cell 16: 777-787. doi:10.1016/j.molcel .2004.11.013
Cioce M, Boulon S, Matera AG, Lamond Al. 2006. UV-induced fragmentation of Cajal bodies. J Cell Biol 175: 401-413. doi:10 $.1083 / j c b .200604099$

Dankert JF, Rona G, Clijsters L, Geter P, Skaar JR, BermudezHernandez K, Sassani E, Fenyo D, Ueberheide B, Schneider R, et al. 2016. Cyclin F-mediated degradation of SLBP limits H2A.X accumulation and apoptosis upon genotoxic stress in G2. Mol Cell 64: 507-519. doi:10.1016/j.molcel.2016 .09 .010

Dominguez-Sanchez MS, Barroso S, Gomez-Gonzalez B, Luna R, Aguilera A. 2011. Genome instability and transcription elongation impairment in human cells depleted of $\mathrm{THO} /$ TREX. PLoS Genet 7: e1002386. doi:10.1371/journal.pgen 1002386

Erkmann JA, Sanchez R, Treichel N, Marzluff WF, Kutay U. 2005. Nuclear export of metazoan replication-dependent histone mRNAs is dependent on RNA length and is mediated by TAP. RNA 11: 45-58. doi:10.1261/rna.7189205

Giacometti S, Benbahouche NEH, Domanski M, Robert MC, Meola N, Lubas M, Bukenborg J, Andersen JS, Schulze WM, Verheggen C, et al. 2017. Mutually exclusive CBC-containing complexes contribute to RNA fate. Cell Rep 18: 2635-2650. doi:10.1016/j.celrep .2017.02.046

Hamm J, Mattaj IW. 1990. Monomethylated cap structures facilitate RNA export from the nucleus. Cell 63: 109-118. doi:10.1016/ 0092-8674(90)90292-M

Izumi H, McCloskey A, Shinmyozu K, Ohno M. 2014. p54nrb/NonO and PSF promote $U$ snRNA nuclear export by accelerating its export complex assembly. Nucleic Acids Res 42: 3998-4007. doi:10.1093/nar/gkt1365

Jackson SP, Bartek J. 2009. The DNA-damage response in human biology and disease. Nature 461: 1071-1078. doi:10.1038/ nature08467

Ji X, Zhou Y, Pandit S, Huang J, Li H, Lin CY, Xiao R, Burge CB, Fu XD. 2013. SR proteins collaborate with 7SK and promoter-associated nascent RNA to release paused polymerase. Cell 153: 855-868. doi:10.1016/j.cell.2013.04.028

Kiriyama M, Kobayashi Y, Saito M, Ishikawa F, Yonehara S. 2009. Interaction of FLASH with arsenite resistance protein 2 is involved in cell cycle progression at S phase. Mol Cell Biol 29: 4729-4741. doi:10.1128/MCB.00289-09

Kohler A, Hurt E. 2007. Exporting RNA from the nucleus to the cytoplasm. Nat Rev Mol Cell Biol 8: 761-773. doi:10.1038/ nrm2255

Lemm I, Girard C, Kuhn AN, Watkins NJ, Schneider M, Bordonne R, Luhrmann R. 2006. Ongoing $U$ snRNP biogenesis is required for the integrity of Cajal bodies. Mol Biol Cell 17: 3221-3231. doi:10.1091/mbc.e06-03-0247

Machitani M, Taniguchi I, Ohno M. 2020. ARS2 regulates nuclear paraspeckle formation through $3^{\prime}$-end processing and stability of NEAT1 long noncoding RNA. Mol Cell Biol 40: e00269-19. doi:10.1128/MCB.00269-19

Marzluff WF, Wagner EJ, Duronio RJ. 2008. Metabolism and regulation of canonical histone mRNAs: life without a poly(A) tail. Nat Rev Genet 9: 843-854. doi:10.1038/nrg2438

Masuyama K, Taniguchi I, Kataoka N, Ohno M. 2004. RNA length defines RNA export pathway. Genes Dev 18: 2074-2085. doi:10 $.1101 / \mathrm{gad} .1216204$

McCloskey A, Taniguchi I, Shinmyozu K, Ohno M. 2012. hnRNP C tetramer measures RNA length to classify RNA polymerase II transcripts for export. Science 335: 1643-1646. doi:10.1126/science .1218469

Ohno M, Segref A, Bachi A, Wilm M, Mattaj IW. 2000. PHAX, a mediator of $U$ snRNA nuclear export whose activity is regulated by 
phosphorylation. Cell 101: 187-198. doi:10.1016/S0092-8674(00) 80829-6

Ohno M, Segref A, Kuersten S, Mattaj IW. 2002. Identity elements used in export of mRNAs. Mol Cell 9: 659-671. doi:10.1016/ S1097-2765(02)00454-9

Seluanov A, Mao Z, Gorbunova V. 2010. Analysis of DNA doublestrand break (DSB) repair in mammalian cells. J Vis Exp 2002. doi:10.3791/2002

Shrivastav M, De Haro LP, Nickoloff JA. 2008. Regulation of DNA double-strand break repair pathway choice. Cell Res 18: 134-147. doi:10.1038/cr.2007.111
Thiriet C, Hayes JJ. 2005. Chromatin in need of a fix: phosphorylation of H2AX connects chromatin to DNA repair. Mol Cell 18: 617-622. doi:10.1016/j.molcel.2005.05.008

Will CL, Luhrmann R. 2001. Spliceosomal UsnRNP biogenesis, structure and function. Curr Opin Cell Biol 13: 290-301. doi:10.1016/ S0955-0674(00)00211-8

Zhao J, Kennedy BK, Lawrence BD, Barbie DA, Matera AG, Fletcher JA, Harlow E. 2000. NPAT links cyclin E-Cdk2 to the regulation of replication-dependent histone gene transcription. Genes Dev 14: 2283-2297. doi:10.1101/gad .827700 

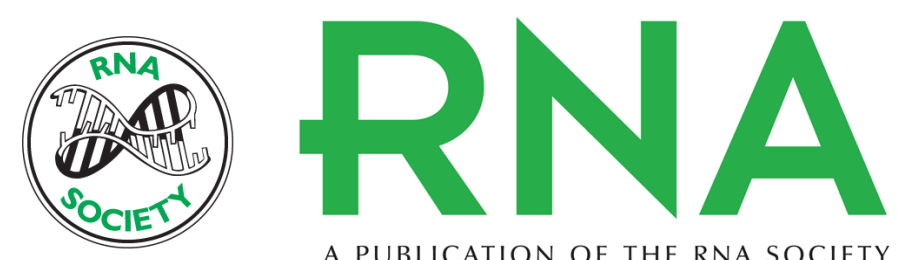

A PUBLICATION OF THE RNA SOCIETY

\section{The RNA transport factor PHAX is required for proper histone H2AX expression and DNA damage response}

Mitsuhiro Machitani, Ichiro Taniguchi, Asako McCloskey, et al.

RNA 2020 26: 1716-1725 originally published online August 5, 2020

Access the most recent version at doi:10.1261/rna.074625.120

\section{Supplemental http://rnajournal.cshlp.org/content/suppl/2020/08/05/rna.074625.120.DC1 \\ Material}

References This article cites 27 articles, 8 of which can be accessed free at: http://rnajournal.cshlp.org/content/26/11/1716.full.html\#ref-list-1

Creative This article is distributed exclusively by the RNA Society for the first 12 months after the Commons

License full-issue publication date (see http://rnajournal.cshlp.org/site/misc/terms.xhtml). After 12 months, it is available under a Creative Commons License (Attribution-NonCommercial 4.0 International), as described at http://creativecommons.org/licenses/by-nc/4.0/.

Email Alerting Receive free email alerts when new articles cite this article - sign up in the box at the Service top right corner of the article or click here.

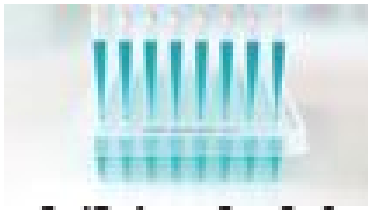

Providing Precise Solutions for your research.

To subscribe to $R N A$ go to:

http://rnajournal.cshlp.org/subscriptions

(C) 2020 Machitani et al.; Published by Cold Spring Harbor Laboratory Press for the RNA Society 